\title{
Development of a dot-ELISA for detection of leprosy antigenuria under field conditions
}

\author{
RASHAD-RUDOLF J. KALDANY \& AYENEW NURLIGN \\ Armauer Hansen Research Institute, Addis Ababa, Ethiopia
}

\begin{abstract}
A dot-ELISA using nitrocellulose paper as the support has been developed to detect phenolic glycolipid-1 under field conditions. Urine from healthy individuals and those diagnosed as having leprosy were concentrated 100 times using a combination of ultrafiltration and lyophilization. $100 \mu \mathrm{l}$ aliquots were treated with $5 \%$ TCA, or extracted with chloroform/ methanol (2:1), or directly applied to the nitrocellulose filter. The antigen was detected using a mouse monoclonal antibody. PGL-1 was most easily detected in the urine of lepromatous leprosy patients following extraction with chloroform/methanol. PGL-1 was detected in patients' urine for up to 2 months after the onset of multidrug therapy.
\end{abstract}

\section{Introduction}

Case detection and treatment are the principal methods currently used for the control of leprosy. Despite apparently effective chemotherapy since the 1940's, the prevalence rate of leprosy in certain regions remains relatively high-over 10 per 1000 . There are many factors which contribute to this persistently high prevalence rate, but case detection may be one of the most critical. In one study, it was estimated that less than half of the 11.5 million cases world-wide (1) of clinical leprosy are actually registered (2).

The advent of multidrug therapy promises a relatively rapid decrease in the prevalence rate of leprosy. Epidemiological evidence suggests that human infections are the most important, if not the only, source of infection in man. It is tempting to speculate that if one could only detect all leprosy cases, eradication of the disease would soon be at hand.

Efforts to achieve effective case-detection can be thwarted by many factors. The social stigma associated with the disease, geographical isolation of affected individuals, and the imperfection or impracticality of available diagnostic tools are but a few factors. Recent efforts to develop reliable diagnostic assays suitable for field work promise to facilitate the field workers' task of case detection.

We describe here our latest efforts to develop a simple, inexpensive, and reliable diagnostic assay for leprosy. In a previous communication (3), it was reported that the Mycobacterium leprae specific phenolic glycolipid-1 (PGL-1) was present in detectable amounts in the urine of leprosy patients. This was determined by an inhibition ELISA using a synthetic analog of PGL-1 as the substrate and leprosy patients' serum as a source of antibody against PGL-1. The inhibition of this reaction was compared with the inhibition resulting from the addition of known amounts of a sonicated suspension of PGL-1 in water. The equivalent of $2.5 \mu \mathrm{g}$ of PGL- $1 / \mathrm{ml}$ was detected in the urine of lepromatous leprosy patients. Patients in the more tuberculoid end of the disease spectrum had much lower amounts. The sensitivity of this assay was low, requiring more than $0.5 \mu \mathrm{g}$ of PGL- 1 to elicit a detectable amount of in- 
hibition. In a separate study also using an inhibition ELISA, the equivalent of $20-35 \mathrm{ng} / \mathrm{ml}$ of PGL-1 was detected in the urine of one lepromatous leprosy patient (4).

Greater sensitivity in the detection of PGL-1 was achieved through the direct detection on nitrocellulose paper using the dot-ELISA technique; as little as $3 \mathrm{ng}$ of a suspension of PGL-1 in water was easily visualized without special enhancement. PGL-1 was detected in a $3 \mu \mathrm{l}$ aliquot of 100 fold concentrated urine sample from some lepromatous leprosy patients. However, this assay failed to reveal any PGL-1 in the urine of several patients and in several of the urine samples a high non-specific signal was observed which could have obscured the analysis. We report here our efforts to increase the sensitivity of the dot-ELISA while avoiding introducing complexities to the overall procedure which would obviate its use under field conditions.

\section{Materials and Methods PATIENTS}

As described in previous communications $(5,6,7)$, untreated leprosy patients attending the out-patient clinic of the All Africa Leprosy and Rehabilitation Training Centre (ALERT) in Addis Ababa, Ethiopia were selected. The diagnosis was based on the clinical findings, the results of examining slit-skin smears for acid-fast bacilli (Ziehl-Nielsen staining); and, in most cases by histopathological evaluation of skin biopsies using the Ridley-Jopling classification (8).

\section{URINE SAMPLES}

Urine samples (100-300 ml volumes) collected for an earlier study $(5,6,7)$ were used. The samples were first concentrated tenfold by ultrafiltration across a YMl0 (Amicon) membrane with a 10,000 dalton cut-off. The concentrate was then lyophilized and finally reconstituted to $1 / 100$ the original volume in $0.01 \mathrm{M}$ phosphate buffered saline containing $0.2 \%$ bovine serum albumin (BSA) (Sigma Chemical Company) and $0.02 \%$ sodium azide. The five samples tested in this study were drawn randomly from the set used in an earlier study (7) and were restricted to lepromatous leprosy patients.

\section{ANTIGENS}

M. leprae glycolipid antigens were provided by the National Institutes of Health (U.S.A.) through Dr. P.J. Brennan, Colorado State University. Native phenolic glycolipid 1 (PGL-1) and deacylated PGL- 1 were used (9). One $\mathrm{ml}$ of water was added to $1 \mathrm{mg}$ dried antigen preparations that were then exposed to ultrasound from an immersion probe for 1 min to produce a uniform milky suspension. Dilutions of antigen in water were made as appropriate.

\section{TRICHLOROACETIC ACID AND/OR HEAT TREATMENT OF URINE SAMPLES}

Aliquots $(200 \mu \mathrm{l})$ of a suspension of PGL-1 $(0.2 \mu \mathrm{g} / \mathrm{ml})$ in water or in 100 -fold concentrated urine were boiled $\left(92^{\circ} \mathrm{C}\right)$ for $5 \mathrm{~min}$, or brought to $5 \%$ trichloroacetic acid and cooled for 5 min in an ice bath, or subjected to a combination of the two treatments. The trichloroacetic acid treated samples were centrifuged at 17,000xg for $5 \mathrm{~min}$ in an Eppendorf microfuge and the supernatant then aspirated and neutralized with $10 \mathrm{~N} \mathrm{NaOH}$ and $1 \mathrm{M}$ Tris. $\mathrm{Cl}(\mathrm{pH} \mathrm{8.0)}$ buffer. The samples were divided into two equal parts and applied to nitrocellulose paper.

\section{EXTRACTION OF URINE SAMPLES WITH CHLOROFORM/METHANOL}

Aliquots $(200 \mu \mathrm{l})$ of a suspension of PGL-1 $(0.2 \mu \mathrm{g} / \mathrm{ml})$ in water or in urine were added to 1 $\mathrm{ml}$ of a 2:1 mixture of chloroform/methanol. After vigorously mixing by hand the two phases, the samples were centrifuged for $30 \mathrm{sec}$ at 17,000xg. The lower phase was removed and 
placed in a second Eppendorf tube; the volatile solvents were dried under vacuum. Phosphate buffered saline $(500 \mu \mathrm{l})$ was added; samples were subjected to sonication ( 80 watts, Branson Sonifier Model B 12) for $30 \mathrm{sec}$, divided into two equal parts and then applied to the nitrocellulose paper.

\section{DOT-BLOT ASSAY}

Pre-washed nitrocellulose filter (Sartorius-0.2 $\mu \mathrm{m}$ and $0.45 \mu \mathrm{m}$ ) was allowed to air-dry before being installed in a Bio-Dot microfiltration apparatus (Bio-Rad). In each experiment, half of each sample of urine or solutions of PGL-1 was applied to a separate well. Vacuum was applied; subsequent to filtration of the sample, blocking of sites not bound with antigen was effected with $1 \% \mathrm{BSA} / 0.5 \%$ gelation in $10 \mathrm{mM}$ Tris $(\mathrm{pH} 7.4,150 \mathrm{mM} \mathrm{NaCl}$ (Standard Dot-Blot Buffer or DSB Buffer) for $30 \mathrm{~min}$. at room temperature. The filter was then cut into strips such that each of the duplicate aliquots could be incubated with PGL-1 at a concentration of $25 \mu \mathrm{g} / \mathrm{ml}$. The primary antibody, mouse monoclonal antibody, was diluted 20,000 fold in SDB buffer and incubated with the filter overnight at room tempertaure. The filter was rinsed three times with SDB buffer and then incubated with peroxidase-conjugated rabbit anti-mouse (Dakopatts) diluted 100 fold for at least two hours. After washing three times with SDB, the bound antibodies were visualized with 4-chloro-1-naphthol (Sigma) at a concentration of $10 \mathrm{mg}$ per $50 \mathrm{ml}$ or $10 \mathrm{mM}$ Tris $(\mathrm{pH} 7.4)$ in $100 \mathrm{mM} \mathrm{NaCl}$.

\section{ANTIBODY}

Mouse monoclonal antibody against PGL-1 was provided by the Center for Disease Control. The IMMLEP designation is MC-1433 (10). The antibody preparation was diluted 20,000 fold prior to use.

\section{Results}

\section{HEAT AND TRICHLOROACETIC ACID TREATMENT OF URINE SAMPLES}

The effect of subjecting PGL- 1 to elevated temperatures $\left(92^{\circ} \mathrm{C}\right)$ or to exposure to high concentrations of acid was tested (Figure 1). This treatment did not appear to alter the immunogenic moiety of PGl-1 (Figure 1); the intensity of the dot was approximately equal in all the conditions when $0.2 \mu \mathrm{m}$ pore size paper was used (see spots $\mathrm{Cl}-8$ in Figure 1). When 0.45 $\mu \mathrm{m}$ paper was used the PGL-1 appears not to have been bound very efficiently except in the presence of high salt concentrations as in the samples in which trichloroacetic acid and the neutralizing salts were added (see spots A3, 4,7 and 8 in Figure 1). A urine sample from a lepromatous leprosy patient was similarly subjected to heat and/or treatment. When neither heat nor trichloroacetic acid was added, a small signal could be detected when compared to the duplicate dot in which the binding of the anti-PGL-1 binding was blocked by excess exogenous PGL-1. Heat treatment alone decreased the signal by several fold while also decreasing the background. A combination of heat and trichloroacetic acid had a lower signal than trichloroacetic alone but was higher than no treatment.

\section{EXTRACTION OF PGL-1 FROM URINE WITH CHLOROFORM/METHANOL}

The amount of PGL-1 extracted by chloroform/methanol (spot A2-Figure 2) was compared to a sample of PGL-1 to which chloroform/methanol was added, mixed, and the dried (spot A 1-Figure 2). The extraction appeared to be quantitative and the same was observed when a PGL-1 suspension was added concentrated urine (spot A3-Figure 2). Concentrated samples of urine from four lepromatous leprosy (LL) individuals (spot A4, 5, 6 and 8) and one borderline lepromatous (BL) individual (spot A7) were similarly extracted with chloroform/ methanol. All four LL patients had easily detectable spots whereas the BL patient was faint. 

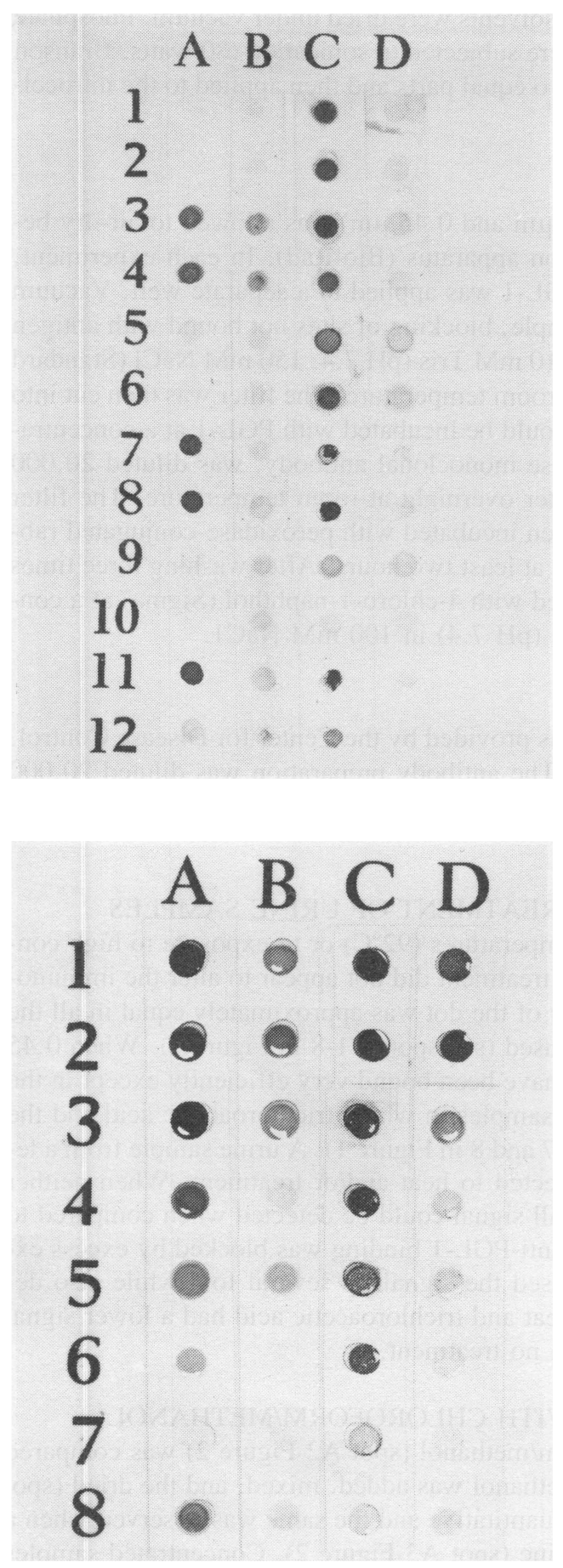

Figure 1.

The effect of heat and acid treatment on the detection PGL-I in urine: PGL-I (l ug) was added to 200 ul of water (rows 1-4) or urine (rows 5-8) from untreated patients 103 (7). Rows 9-12 consisted of 200 ul of urine from untreated patient 103 (7). Rows 1, 5, and 9 had not treatment; rows 2, 6, and 10 were heated at $92^{\circ} \mathrm{C}$ for $5 \mathrm{~min}$; rows 3,7 , and 11 were brought to $5 \%$ trichloroacetic acid; rows 4, 8, and 12 were boiled for $5 \mathrm{~min}$ at $92^{\circ} \mathrm{C}$ brought to $5 \%$ trichloroacetic acid and the processed as in described in the methods section. Lanes $A$ and $C$ were incubated with the monoclonal antibody and lanes $B$ and $D$ were incubated with the monoclonal antibody and lanes $B$ and $D$ were incubated with the nomoclonal antibody in the presence of $25 \mathrm{ug} / \mathrm{ml}$ of PGL 1.

Figure 2.

Extraction of PGL-1 from urine samples with chloroform/methanol: PGL-1 (0.5 ug) was added to 200 ul of water (rows 1 and 2) or to urine (spots $A 3$ and B3) from untreated patient 115 (reference 7). Row 1 samples had chloroform/methanol added but were not extracted. All other samples were extracted as described in methods. Urine from the following patients (reference7) were spotted in adjacent wells: untreated 103 (A4 and B4), untreated 121 (A5 and B5), untreated 120 (A6 and $B 6)$, untreated 119 (A7 and $B 7)$, and 4 week after onset of treatment 115 (A8 and B8). Lanes $C$ and D contained a series of samples from patient 103 before and after onset of treatment as follows: $2 \mathrm{da}$ ys before (row 3), I day before (row 4), 3 days after (row 5), 2 weeks after, 3 weeks after (row 7), and 2 months after (row 8). Lanes $A$ and $B$ used 0.45 um and lanes $C$ and $D$ used 1.2 um nitrocellulose filters. 
In a separate experiment, urine from a patient before and after onset of treatment was analyzed for PGL- 1 content. The amount of PGL- 1 increased after 1 week and then rapidly decreased after two months of treatment with dapsone, rifampicin, clofazimine, and ethionamide.

In one experiment, one patient's urine, known to produce a positive signal in the dotELISA, was diluted to its original volume $(20 \mathrm{ml})$ before extracting with chlorof $\mathrm{orm} / \mathrm{meth}$ anol $(1 \mathrm{ml})$. The extract was treated as before and the resuspended material analyzed by the dot-ELISA. The amount of PGL-1 detected was found to be greater than in the concentrated urine sample (data not shown).

We have subjected serum samples to the same treatment; however, we have not succeeded in detecting PGL-1 in any samples.

\section{Discussion}

In an earlier communication, the direct detection of PGL- 1 using a dot-ELISA was reported. The urine samples used as source material were concentrated 100 fold using a combination of ultrafiltration and lyophilization. The urine samples were directly analyzed using dot-ELISA. In many of the urines, the background signal appeared to obscure the specific signal due to PGL-1. Several approaches were thus considered to decrease the background noise and to increase the signal. The two approaches consisted of doing simple partial purifications either by precipitating non-PGL-1 components or, alternatively, extracting it.

The presence of antibodies in the urine of leprosy patients may be masking the same epitopes on PGL-1 which we are trying to detect. We therefore attempted to dissassociate the PGL-1 from the antibodies by subjecting the urine to high heat, to high concentrations of acid, or to a combination of both. We found that neither heat nor high concentrations of trichloroacetic acid affected the immunogenic moiety of PGL-1. The trichloroacetic acid treatment resulted in an enhancement in the amount of PGL-1 detected in several urine samples. This may be attributed to the acid's ability to differentially precipitate components in the urine which gave high backgrounds and which also may have more directly inhibited the PGL-1 from binding to the nitrocellulose filter. However, in urine samples that appeared to contain very large amounts of precipitable components no enhancement in sensitivity was observed. The hydrophobic interaction of the PGL-1 with precipitable components may be responsible for this observation.

Extraction of the PGL-1 with a mixture of chloroform/methanol has proved to be the most effective sample preparation when judged by the signal observed in the presence and absence of exogenous PGL- 1 during incubation with the primary antibody. In addition, this method promises to be the simplest method. In one experiment, an unconcentrated urine sample was extracted with the organic solvents and resulted in a very strong signal. The advantage of this technique is, therefore, that sample prepation is reduced to a minimum.

Two other methods to detect PGL-1 in serum $(4,11)$ and urine $(4)$ have been reported. In one method (11), the PGL-1 is extracted, partially purified on silicic acid and celite, and then separated on polysulfone membrane. In the other (4), the PGL-1 is extracted and then purified through several steps. Although these techniques are very sensitive, they are, unfortunately, too complicated for application under field conditions. The aim of our project has been to develop techniques which can be relatively easily adapted for field work. For instance, centrifugation of urine samples to which trichloroacetic acid has been added can be accomplished using a hand-driven centrifuge. In the case of the extraction procedure, heating to $40^{\circ} \mathrm{C}$ could increase the rate of evaporation of the solvents, thus obviating the need for a vacuum pump. Finally, instead of using a manifold, which also requires a vacuum pump, large volumes of urine or resuspended material can be filtered directly in a syringe. The fea- 
sibility of this technique has been shown using a $1 \mathrm{~cm}$ in diameter nitrocellulose filter attached to the end of a syringe. Thus, 10 ng of PGL-1 in water or in urine in volumes as large as $5 \mathrm{ml}$ were easily detected on the filter.

The techniques described here are still not sensitive enough to detect antigen in patients in the more tuberculoid end of the disease spectrum. However, they are simple enough to be performed on a routine basis and can be adapted for field work and, it is hoped, that further improvements in sensitivity and selection of novel antigens may result in an assay which can detect antigenuria even in paucibacillary patients.

\section{Acknowledgements}

The authors would like to thank Dr. P.J. Brennan for his generous gifts of reagents. The critical comments and suggestions of Paul Converse and Rune Nilsen are gratefully acknowledged. This research was supported by Redd Barna and Rädda Barnen (Norwegian and Swedish Save the Children) and NIH grant 20198.

\section{References}

1 Sansarricq H. Recent changes in leprosy control. Leprosy Review, 1981, special issue, 7-16.

2 Report of a meeting on action plan for leprosy control. New Delhi, 23-25 August 1982. Unpublished WHO document WHO/LEP/83.1 Corr. 1.

3 Kaldany R-R J, Maasho K, Ohman R, Reitz-Vick D, Britton S, Lefford MJ. Methods for the detection of a specific Mycobacterium leprae antigen in the urine of leprosy patients. Scand. J. Immunol., 1987, 25: 37.

4 Cho S-N, Hunter SW, Gelber RH, Rea TH, Brennan PJ. Quantitation of the phenolic glycolipid of Mycobacterium leprae and relevance to glycolipid antigenemia in leprosy. J. Infect. Dis., 1986, 153: 560-569.

5 Olcen P, Harboe M, Warndorff T, Belehu A. Antigens of Mycobacterium leprae and anti-Mycobacterium leprae antibodies in the urine of leprosy patients. Lepr. Rev., 1983; 54: 203-216.

6 Olcen P, Harboe M, Warndorff T, Belehu A. Anti-Mycobacterium leprae antibodies in urine from lepromatous patients examined by crossed immunolectrophoresis and radioimmunoassay, Scand. J. Immunol. 1984; 19: 521-528.

7 Olcen P, Harboe M, Warndorff T. Antigens of Mycobacterium leprae in urine during treatment of patients with lepromatous leprosy. Lepr. Rev., in press.

8 Ridley DS, Jopling WH. Classification of leprosy according to immunity. A five-group system. Int. J. Lepr. 1966, 34: 255-73.

9 Hunter SW, Fujiwara T, Brennan PJ. Structure and antigenicity of the major specific glycolipid antigen of Mycobacterium leprae. J. Biol. Chem., 1982; 257: 15072-8.

10 Engers HD, Bloom BR, Godal T. Mocnolonal antibodies against mycobacterial antigens. Immunology Today 1985; 6: 345-8.

11 Young DB, Fohn MJ, Buchanan TN. Use of a polysulfone membrane support for immunochemical analysis of a glycolipid from Mycobacterium leprae. J. Immunol. Methods 1985; 79: 205-11. 